ISSN 0103-5150

Fisioter. Mov., Curitiba, v. 26, n. 3, p. 623-629, jul./set. 2013

Licenciado sob uma Licença Creative Commons

\title{
0 método Pilates no tratamento de espondilolistese traumática em L4-L5: estudo de caso
}

\author{
The Pilates method in the treatment of traumatic \\ spondylolisthesis at L4-L5: case report
}

\section{Laís Campos de Oliveira ${ }^{[a]}$, Camila da Silva Hoshina ${ }^{[b]}$, Larissa Alexandre Furlan ${ }^{[c]}$, Raphael Gonçalves de Oliveiraa ${ }^{[\mathrm{d}]}$, Fábio Antônio Néia Martini ${ }^{[\mathrm{e}]}$}

[a] Fisioterapeuta, especialista em Fisioterapia Traumato-Ortopédica pela Universidade Gama Filho (UGF), aluna do programa de mestrado em Exercício Físico na Promoção da Saúde da Universidade Norte do Paraná (Unopar), Londrina, PR - Brasil, e-mail: laispiraju@yahoo.com.br

[b] Fisioterapeuta, aluna do curso de especialização em Fisioterapia Ortopédica, Traumatológica e Desportiva pela Faculdade Sudoeste Paulista (FSP), Avaré, SP - Brasil, e-mail: milinhahoshina@hotmail.com

[c] Fisioterapeuta, especialista em Fisioterapia Dermatofuncional pela Universidade Gama Filho (UGF), Rio de Janeiro, RJ Brasil, e-mail: lalifurlan19@hotmail.com

[d] Mestre em Educação Física pela Universidade Metodista de Piracicaba (Unimep), docente do Centro de Ciências da Saúde da Universidade Estadual do Norte do Paraná (Uenp), Jacarezinho, PR - Brasil, e-mail: rgoliveira@uenp.edu.br

[e] Fisioterapeuta, doutor em Ciências do Desporto, diretor e docente do Centro de Ciências da Saúde da Universidade Estadual do Norte do Paraná (Uenp), Jacarezinho, PR - Brasil, e-mail: fabiomartini@uol.com.br

\section{Resumo}

Introdução: 0 método Pilates é uma forma de tratamento conservador, que vem sendo utilizado em patologias da coluna vertebral. Dentre estas se encontra a espondilolistese, que se mostra como uma ocorrência rara, quando do tipo traumática entre as vértebras L4-L5. Objetivo: Verificar os efeitos do método Pilates sobre força, flexibilidade e dor de um paciente com espondilolistese traumática em L4-L5, que apresentava quadro estável. Materiais e métodos: Avaliações pré e pós-intervenção foram realizadas com o intuito de avaliar a força da musculatura abdominal e paravertebral, a flexibilidade da cadeia posterior e a dor lombar, envolvendo os seguintes testes: enrolamento repetitivo do tronco, estático de resistência das costas de Sorenson, sentar e alcançar e escala visual analógica (EVA) da dor. Para a intervenção, foram realizados exercícios do método Pilates, uma hora por dia, quatro vezes na semana, durante 12 semanas, nesta sequência: alongamentos iniciais, fortalecimento de membros inferiores, 
abdominais e membros superiores, alongamentos finais e relaxamento. Resultados: 0 paciente passou de 28 para 39 repetições no teste de enrolamento repetitivo do tronco, e de 17 para 65 segundos, no teste estático de resistência das costas de Sorenson. No teste de sentar e alcançar passou de -5 para $2 \mathrm{~cm}$ e o nível de dor, de 7 para 3. Conclusão: 0 método Pilates se mostrou eficiente no aumento da força da musculatura abdominal e paravertebral, da flexibilidade da cadeia posterior e na melhora da dor na coluna lombar, em um paciente com espondilolistese traumática de L4-L5, sendo uma alternativa para o tratamento conservador, em casos em que não exista instabilidade no quadro.

Palavras-chave: Espondilolistese. Traumatismos da coluna vertebral. Fisioterapia.

\section{Abstract}

Introduction: The Pilates method is a form of conservative treatment, which has been used in diseases of the spine. Among the diseases is spondylolisthesis, which is a rare event when the type traumatic between the L4-L5 vertebrae. Objective: Investigate the effects of Pilates on the strength, flexibility and pain, in a patient with traumatic spondylolisthesis at L4-L5 stable. Materials and methods: Pre- and post-intervention were performed to assess the strength of the abdominal muscles and posterior chain flexibility and low back pain, involving the following tests: repeated rolling of the trunk, static endurance of the back of Sorenson, sit and reach and scale visual analogue score (VAS) of pain. For the intervention, were made of the Pilates exercises, one hour per day, four times a week for twelve weeks, in the following sequence: the initial stretching, strengthening of the lower limbs, abdomen and upper limbs, stretching and final relaxation. Results: Patient went from 28 to 39 repetitions in the test repeated rolling of the trunk and 17 to 65 seconds in the static test of endurance of the back of Sorenson. In the sit and reach test increased from -5 to $2 \mathrm{~cm}$ and the pain level of 7 to 3. Conclusion: The Pilates method was proved effective in increasing muscle strength and abdominal paravertebral, posterior chain flexibility and to reduce pain in the lumbar spine in a patient with traumatic spondylolisthesis of L4-L5, being an alternative to conservative treatment in cases where there is not instability.

Keywords: Spondylolisthesis. Spinal injuries. Physical therapy.

\section{Introdução}

0 método Pilates vem sendo utilizado no tratamento de patologias relacionadas à coluna vertebral, trazendo entre outros benefícios a melhora das funções e da dor do paciente $(1,2)$. Por meio de exercícios que podem ser realizados no solo, com auxílio de acessórios como bola, halteres e caneleiras, é possível proporcionar aumento da força dos músculos estabilizadores da coluna, melhora da flexibilidade da cadeia posterior e maior resistência muscular do corpo como um todo (3); estes são fatores importantes a serem recuperados em pacientes com patologias na coluna (4).

Uma das patologias que podem acometer a coluna vertebral é a espondilolistese, que se caracteriza pelo deslizamento anterior de um corpo vertebral sobre a vértebra subjacente. A do tipo traumática é uma forma rara, principalmente na coluna lombar, causa dor e prejudica funções como força e flexibilidade; é comumente tratada por intervenção cirúrgica (5-8). No entanto, em alguns casos de espondilolistese, dependendo da estabilidade do quadro, o tratamento convencional é indicado $(4,9)$.

Este estudo tem o intuito de verificar os efeitos do método Pilates sobre a força, a flexibilidade e a dor em paciente com espondilolistese traumática de L4-L5 que apresentava quadro estável.

\section{Métodos}

Realizou-se estudo de caso com um paciente do sexo masculino, 41 anos de idade, $58 \mathrm{~kg}$ e 1,65 m, casado, morador de uma cidade do interior do estado do Paraná, Brasil. Essa pessoa apresentou espondilolistese decorrente de uma queda sofrida em agosto de 2000; o diagnóstico foi efetuado na ocasião, em um hospital, e foi identificada como do tipo traumática de grau 2, que corresponde ao deslocamento de dois 
quartos da vértebra superior sobre a inferior (10), no caso, L4 sobre L5 (Figura 1).

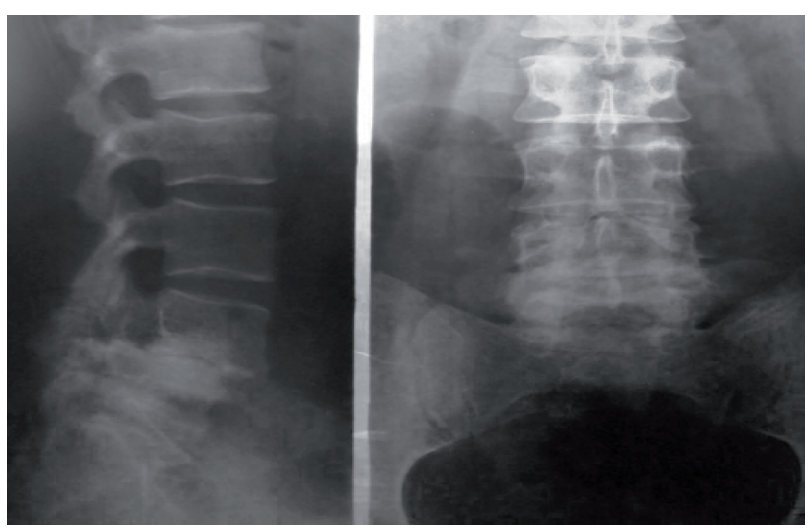

Figura 1 - Radiografia em anteroposterior e perfil demonstrando espondilolistese traumática de L4-L5 em grau 2, realizada antes da intervenção com 0 método Pilates em agosto de 2010

Fonte: Dados da pesquisa.

Devido à estabilidade do quadro, o paciente foi submetido nos anos subsequentes a tratamentos conservadores, mais especificamente à técnica de Hidroterapia; esse processo refletiu em oscilações de melhora da dor, mas sem alteração do grau de deslizamento vertebral.

Tendo em vista as queixas de dores na região lombar e limitações em alguns movimentos que exigiam força e flexibilidade, em julho de 2010, o voluntário foi convidado a participar da presente pesquisa, realizada na Clínica de Fisioterapia do Centro de Ciências da Saúde, da Universidade Estadual do Norte do Paraná (Uenp), câmpus Jacarezinho (PR). A instituição autorizou a realização da pesquisa, que foi aprovada no Comitê de Ética, parecer n, 57/2010 e o voluntário assinou Termo de Consentimento Livre e Esclarecido, conforme determina a Resolução 196/96 do Conselho Nacional de Saúde.

O indivíduo era iniciante no método Pilates e não realizou outro tipo de intervenção fisioterapêutica durante o tratamento.

\section{Avaliação}

Antes e após a intervenção, o paciente realizou radiografias em uma visão anteroposterior e perfil da região lombar, na intenção de observar se o grau de deslizamento vertebral ainda se encontrava em grau 2 , o que foi identificado nas duas ocasiões. Para análise do grau de deslizamento da vértebra, foi usado o sistema de classificação em categorias de Meyerding (10). Analisando a radiografia em uma visão de perfil, o grau 1 corresponde ao deslizamento anterior da vértebra superior, até um quarto da vértebra inferior, de modo que o grau aumenta para cada quarto da dimensão anteroposterior descoberto pela vértebra superior. 0 grau 5 é o mais acentuado, ocorrendo um deslocamento completo da vértebra superior.

A respeito da instabilidade na coluna, a literatura ainda é contraditória. Comumente se utiliza de radiografias com o paciente na posição ortostática, realizando os movimentos de flexão e extensão da coluna (para verificar se existe um deslocamento adicional igual ou maior que $4 \mathrm{~mm}$, ou diferença na mobilidade angular acima de 11 graus em resposta a flexão e extensão) ou testes específicos para avaliar o conjunto dos seguintes sintomas: dor insuportável, deficit neurológico e claudicação (11).

Optou-se pela segunda forma de avaliação, empregando-se os seguintes testes: escala visual analógica (EVA) da dor (foi considerado dor insuportável o nível de 8 a 10) (12), reflexo patelar e Aquileu, teste de sensibilidade (tátil, térmico e doloroso), teste de marcha, teste de coordenação e equilíbrio (13). Ao ser constatado que o paciente ainda se encontrava com deslizamento em grau 2 e com estabilidade no quadro, foram realizadas as avaliações descritas na sequência.

Os testes de enrolamento repetitivo do tronco e estático de resistência das costas de Sorenson foram realizados, respectivamente, para avaliar a força dos músculos abdominais e dos paravertebrais, dois dos músculos importantes para a estabilização da coluna lombar, seguindo as orientações de Cox (13).

No teste de enrolamento repetitivo do tronco, o paciente, em decúbito dorsal, joelhos flexionados a 90 graus e tornozelos fixos apoiados pelo avaliador, realizou flexão da coluna (movimento de abdominal), com os braços estendidos, até que a eminência tênar alcançasse a patela, para posteriormente desenrolar até a posição inicial. 0 número de abdominais foi contado até que o paciente não fizesse o movimento de forma correta. Para o segundo teste, o voluntário se encontrava em decúbito ventral sobre uma maca, com a crista ilíaca anterossuperior e o tronco para fora da mesma, mantendo os braços cruzados no peito, com os tornozelos e as coxas apoiadas pelo avaliador. 0 paciente tentou manter seu corpo completamente estendido (posição neutra) pelo maior 
tempo possível; o cronômetro foi desativado quando o avaliado saiu da posição inicial (flexionou o tronco) ou sentiu dores nas costas.

O teste de sentar e alcançar de Wells e Dillon (14) foi realizado para avaliar a flexibilidade da coluna vertebral e dos músculos isquiotibiais (cadeia posterior), os quais estão associados à maioria das queixas dolorosas na região lombar. 0 paciente se encontrava sentado em frente ao banco de Wells (uma caixa de madeira que avalia em centímetros o encurtamento de cadeia posterior), descalço, com os pés apoiados na caixa, os joelhos completamente estendidos, a planta dos pés totalmente em contato com a caixa e ligeiramente afastados. $\mathrm{O}$ avaliador apoiou os joelhos do avaliado na tentativa de assegurar que estes permanecessem devidamente estendidos durante o movimento.

Para registro dos resultados, o avaliado se encontrava com a palma das mãos posicionadas uma sobre a outra, voltadas para baixo e a ponta dos dedos coincidindo; deveria flexionar o tronco de forma que suas mãos deslizassem sobre a caixa, com escala de medida em centímetros, procurando alcançar a maior distância possível em movimento lento e sem solavancos. Foram realizadas três tentativas, anotando-se a melhor delas e em cada oportunidade, a distância alcançada foi mantida por aproximadamente 2 segundos. Considerou-se como ponto zero o local de contato dos pés com a caixa, sendo possível, assim, obter valores negativos.

A avaliação da dor na coluna lombar foi realizada por meio da EVA, seguindo as orientações de Willestaedt, Levander e Hult (12). Foi apresentada ao paciente uma tabela contendo uma reta com os números de zero a 10; explicou-se a ele que zero significava ausência de dor e 10 representava dor de intensidade máxima, ou insuportável. Posteriormente, o paciente assinalou sua percepção de dor.

Todas as avaliações foram realizadas pela mesma fisioterapeuta, sendo uma antes de iniciada a intervenção com o método Pilates e a outra, logo após finalizar tais procedimentos.

\section{Intervenção}

Os exercícios de Pilates foram subdivididos em dois grupos com 20 exercícios cada, sendo o primeiro realizado nas segundas e quartas-feiras e o segundo grupo, nas terças e quintas-feiras, com duração de 1 hora. 0 protocolo de tratamento teve duração de 12 semanas. Foram realizados alongamentos iniciais e finais (três repetições de 20 segundos para cada exercício), fortalecimento de membros inferiores, abdominais e membros superiores (uma série com dez repetições para cada exercício) e relaxamento (cinco minutos de exercício respiratório). Como acessórios, foram utilizados uma bola de $55 \mathrm{~cm}$ (adequada conforme a altura do paciente), faixa elástica (marca Theraband ${ }^{\circledR}$ da cor verde - intensidade forte), colchonete, halteres e caneleiras de 1 e $2 \mathrm{~kg}$.

As recomendações de Craig (15) foram seguidas na orientação dos exercícios e o nome de cada um deles adotado conforme descrição da autora.

0 primeiro grupo de exercícios respeitou a seguinte sequência: (a) alongamentos iniciais - isquiotibiais com faixa elástica, rã, quadril, lateral e dos ombros; (b) fortalecimento de membros inferiores - círculo com as pernas, abaixar e levantar, meia ponta alta e preparação para a ponte; (c) fortalecimento de abdominais - somente contração, abdominal curto, rotação dos oblíquos e cachoeira; (d) fortalecimento de membros superiores - com as mãos levantadas, rosca bíceps, abrace uma árvore e remada; (e) Alongamentos finais - equilibrando a bola e alongamento flexão do tronco; (f) relaxamento - posição de relaxamento um.

Já o segundo grupo de exercícios realizou a seguinte sequência: (a) alongamentos iniciais alongamento de isquiotibiais, sereia e dedos entrelaçados; (b) fortalecimento de membros inferiores - pés afastados, agachamento total, girar a bola, alongando uma perna e ponte completa; (c) fortalecimento de abdominais - contração, mesinha, alongando ambas as pernas e abdominal completo; (d) fortalecimento de membros superiores - cotovelos afastados, isolamento da escápula, continência, concha e serrote; (e) alongamentos finais — dedos entrelaçados e concha; (f) relaxamento - posição de relaxamento dois.

Inicialmente, o paciente realizava os exercícios com o peso do próprio corpo; posteriormente, à medida que progredia durante o tratamento, passou a utilizar halteres e caneleiras como acessórios. A carga era aumentada somente quando o voluntário demonstrava facilidade na execução do movimento. Em todas as sessões era realçada para o paciente a importância de se executar os movimentos segundo os nove princípios do método Pilates, que são: concentração, controle, centragem, respiração, alinhamento postural, leveza, precisão, força e relaxamento (15). 
Todos os exercícios foram orientados por duas fisioterapeutas previamente treinadas no método Pilates.

Por se tratar de um estudo de caso, a análise dos dados foi descritiva, tendo em vista a impossibilidade da realização de análises estatísticas.

\section{Resultados}

Em todos os testes realizados ocorreram melhoras. Para o enrolamento repetitivo do tronco, os valores foram de 28 repetições na avaliação pré-intervenção e 39 na pós-intervenção. No teste estático de resistência das costas de Sorenson, os valores passaram de 17 segundos na avaliação inicial para 65 na avaliação final. Para o teste de sentar e alcançar, inicialmente o paciente atingiu $-5 \mathrm{~cm}$ e na reavaliação $2 \mathrm{~cm}$. Na avaliação da dor (EVA) o voluntário passou do nível 7 para o nível 3.

\section{Discussão}

A espondilolistese foi classificada por Wiltse et al. (16) como degenerativa, ístmica, patológica, displásica e traumática. Essa última é uma forma rara, havendo poucos casos registrados, principalmente quando atinge as vértebras L4-L5, sendo comum encaminhamento para tratamento cirúrgico, tendo em vista a gravidade dos casos (5-8). Uma vez que o quadro do voluntário do presente estudo não apresentava instabilidade, optou-se pelo tratamento conservador, como sugere a literatura $(4,9)$.

O método Pilates é uma forma de tratamento conservador que vem sendo utilizado em vários estudos na área da Fisioterapia $(16,17)$. No caso da espondilolistese, devido à perda de força e diminuição da flexibilidade nos pacientes, o tratamento que envolva recuperação dessas variáveis se torna importante, porque os exercícios de Pilates realizados no solo, com auxílio de acessórios, têm se mostrado efetivos para essa finalidade (3).

Com relação à força, realizando tais exercícios, o voluntário do presente estudo apresentou melhora da musculatura abdominal, com aumento de 11 repetições no teste de enrolamento repetitivo do tronco. Para a musculatura paravertebral, a melhora foi de 48 segundos no teste estático de resistência das costas de Sorenson. Esses músculos auxiliam na estabilização da coluna e estão diretamente relacionados ao princípio da centragem, que, segundo Craig (15), é um dos nove princípios do método Pilates e diz respeito ao fortalecimento da musculatura profunda da região central do corpo, ocorrendo dos músculos mais internos progredindo para os mais externos; a centragem também é conhecida como centro de força, power-house ou core.

Estudos vêm demonstrando a eficácia do método Pilates no fortalecimento dessa região, que envolve os músculos abdominais, paravertebrais lombares e glúteos $(17,18)$. Kloubec $(3)$, ao verificar a força muscular abdominal e paravertebral de pacientes submetidos ao treinamento com o método Pilates, realizados uma hora por dia, dois dias da semana, durante três meses, verificou um aumento significativo de ambas as variáveis. No caso de pessoas com disfunções na coluna lombar, Fonseca et al. (2) constataram que 15 seções de Pilates são capazes de proporcionar melhora significativa da força nessa região.

No que diz respeito à flexibilidade, o paciente obteve uma melhora de $7 \mathrm{~cm}$ no teste de sentar e alcançar, que avalia o alongamento da cadeia posterior do corpo, se aproximando dos dados encontrados por Rogers e Gibson (19). Os autores, ao avaliarem um grupo de adultos saudáveis e iniciantes no método Pilates, observaram um aumento médio de 7,5 $\mathrm{cm}$ nesse mesmo teste, após oito semanas de treinamento, realizados três vezes por semana, durante uma hora por dia.

Nos pacientes com espondilolistese, essa melhora se torna fundamental, uma vez que movimentos simples como se abaixar para pegar um determinado objeto, ou mesmo se levantar da cama, podem ser comprometidos pela perda da flexibilidade (20). Essa variável está diretamente relacionada aos exercícios de Pilates, que ao considerar princípios como controle, alinhamento postural, leveza, precisão, respiração adequada, dentre outros, pode auxiliar no aumento da flexibilidade, o que contribui para a recuperação de movimentos fundamentais para as atividades da vida diária (15).

A melhora da flexibilidade e o fortalecimento dos músculos estabilizadores da coluna têm proporcionado, ainda, a diminuição da dor lombar de pacientes submetidos ao método Pilates $(1,2)$, o que se pode observar também no presente estudo, no qual o voluntário relatou uma melhora de 4 pontos na sua percepção de dor, de acordo com a EVA. Yang et al. (21) concluíram que o fortalecimento dos músculos estabilizadores da coluna e a melhora da flexibilidade possibilitaram redução significativa da dor lombar.

No entanto, França et al. (22) observaram em um grupo de indivíduos com dor lombar crônica que 
aqueles submetidos a exercícios que envolviam estabilização segmentar, como é o caso do método Pilates, relataram uma melhora da dor significativamente maior do que aqueles que realizaram exercícios que promoviam apenas o fortalecimento superficial dos músculos estabilizadores da coluna. Os autores salientaram que se torna necessário priorizar aqueles exercícios em detrimento destes, quando se trata da recuperação de pacientes com dor lombar crônica.

A melhora da dor, como também da força e da flexibilidade, apresentada no presente estudo, sugere que o método Pilates se mostrou uma alternativa para tratamentos conservadores de pacientes com espondilolistese traumática na coluna lombar, que apresentem estabilidade no quadro. No entanto, a literatura ainda é controversa, a respeito das variáveis que determinam a instabilidade. Desse modo, estudos precisam ser realizados para que se estabeleçam parâmetros confiáveis na análise desses casos.

\section{Conclusão}

O método Pilates se mostrou eficiente no aumento da força da musculatura abdominal e paravertebral, melhora da flexibilidade da cadeia posterior e diminuição da dor na coluna lombar em um paciente com espondilolistese traumática de L4-L5, sendo uma alternativa para o tratamento conservador, em casos com quadro estável.

\section{Referências}

1. La Touche R, Escalante K, Linares MT. Treating nonspecific chronic low back pain through the pilates method. J Bodyw Mov Ther. 2008;12(4):364-70.

2. Fonseca JL, Magini M, Freitas TH. Laboratory gait analysis in patients with low back pain before and after a pilates intervention. J Sport Rehabil. 2009;18(2):269-82.

3. Kloubec JA. Pilates for improvement of muscle endurance, flexibility, balance, and posture. J Strength Cond Res. 2010;24(3):661-7.

4. Kalichman L, Hunter DJ. Diagnosis and conservative management of degenerative lumbar spondylolisthesis. Eur Spine J. 2008;17(3):327-35.
5. Lim CT, Hee HT, Liu G. Traumatic spondylolisthesis of the lumbar spine: a report of three cases. J Orthop Surg. 2009;17(3):361-5.

6. Zhou TH, Tang X, Xu YQ Zhu YL. Traumatic spondyloptosis of L4. Spine. 2010;35(17):855-9.

7. Yadla S, Lebude B, Tender GC, Sharan AD, Harrop JS, Hilibrand AS, et al. Traumatic spondyloptosis of the thoracolumbar spine. J Neurosurg Spine. 2008;9(2):145-51.

8. Deniz FE, Zileli M, Cağli S, Kanyilmaz H. Traumatic L4-L5 spondylolisthesis: case report. Eur Spine J. 2008;17(2):232-5.

9. Zoner CS, Amaral DT, Natour J, Fernandes ARC. Contribuição dos métodos de diagnóstico por imagem na avaliação da espondilólise. Rev Bras Reumatol. 2006;46(4):287-91.

10. Resnick D. Diagnosis of bone and joint disorders. 4th ed. Philadelphia: Saunders; 2002.

11. Huang KY, Lin RM, Lee YL, Li JD. Factors affecting disability and physical function in degenerative lumbar spondylolisthesis of L4-5: evaluation with axially loaded MRI. Eur Spine J. 2009;18(12):1851-7.

12. Willestaedt H, Levander G, Hult L. Studies in osteogenesis. Acta Orthop Scand. 1950;19(4):419-32.

13. Cox JM. Low back pain: mechanism, diagnosis, and treatment. 6th ed. Philadelphia: Williams \& Wilkins; 1999.

14. Wells KF, Dillon E. The sit and reach: a test of bank and leg flexibility. Res Q Exerc Sport. 1952;23(1):115-8.

15. Craig C. Pilates com a bola. São Paulo: Phorte; 2005.

16. Wiltse LL, Newman PH, Macnab I. Classification of spondylolisis and spondylolisthesis. Clin Orthop Relat Res. 1976;(117):23-9.

17. Silva ACLG, Mannrich G. Pilates na reabilitação: uma revisão sistemática. Fisioter Mov. 2009;22(3):449-55.

18. Emery K, De Serres SJ, McMillan A, Côté JN. The effects of a pilates training program on arm-trunk posture and movement. Clin Biomech. 2010;25(2):124-30.

19. Rogers K, Gibson AL. Eight-week traditional mat pilates training-program effects on adult fitness characteristics. 2009;80(3):569-74. 
20. Abbott AD, Tyni-Lenné R, Hedlund R. Early rehabilitation targeting cognition, behavior, and motor function after lumbar fusion: a randomized controlled trial. Spine. 2010;35(8):848-57.

21. Yang EJ, Park WB, Shin HI, Lim JY. The effect of back school integrated with core strengthening in patients with chronic low-back pain. Am J Phys Med Rehabil. 2010;89(9):744-54.

22. França FR, Burke TN, Hanada ES, Marques AP. Segmental stabilization and muscular strengthening in chronic low back pain: a comparative study. Clinics. 2010;65(10):1013-17.

Recebido: 29/07/2012

Received: 07/29/2012

Aprovado: 12/03/2013

Approved: 03/12/2013 